\title{
ERRATUM
}

\section{Predicting survival for myeloid leukemia after HLA-identical sibling donor allogeneic stem cell transplantation}

\author{
D Gallardo, R De la Cámara, A Torres, S Brunet, A Jiménez, JC Vallejo, G Sanz, D Serrano, E Carreras, C Martín, \\ C Sanz-Rodríguez, J Sierra, I Espigado, D Caballero and JJ Berlanga
} print containing a Table 1 on page 1033, which is not part of this article. The correct version of the paper is reproduced below.

The Publisher would like to apologise for any inconvenience doi:10.1038/sj.leu.2403344 this may have caused.

\section{CORRESPONDENCE}

\section{Predicting survival for myeloid leukemia after HLA-identical sibling donor allogeneic stem cell transplantation}

\author{
Leukemia (2004) 18, 1031-1034. doi:10.1038/sj.leu.2403344 \\ Published online 18 March 2004

\section{TO THE EDITOR}

Several variables have been previously identified as adverse risk factors for survival after HLA-identical sibling donor allogeneic stem cell transplantation (SCT). Indeed, advanced phase of disease is associated with increased relapse rate and death due to leukemia progression. On the other hand, factors influencing the incidence and severity of graft-versus-host disease (GvHD), such as sex mismatch (female donor and male recipient), older recipient age or positive CMV serology, are related to an increased transplant-related mortality. ${ }^{1,2}$ However, it is sometimes difficult to know the real impact of these risk factors on transplant outcome when considering a single patient, because favorable and adverse conditions may be present at the same time. In this work, we present a clinical score for predicting the survival of allogeneic SCT from HLA-identical sibling donors for myeloid malignancies on the basis of the evaluation and grading of risk factors available pretransplant.

We retrospectively analysed the pretransplant data and the clinical post-transplant outcome for 319 adult patients diagnosed of acute myeloid leukemia (AML) $(n=150)$ or CML $(n=169)$ who received an allogeneic SCT from an HLAidentical sibling donor from 1991 to 2002, performed in 14 centers. Patients with AML were assigned to the following $\mathrm{FAB}$ groups: M0: 3\%; M1: 15\%; M2: 24\%; M3: 6\%; M4: 25\%; M5: 18\%; M6: 3\%; M7: 1\%; post-MDS: 5\%. The median follow-up

Correspondence: Dr D Gallardo, Clinical Hematology Department, Institut Català d'Oncologia, Hospital Durán i Reynals. Avda, Gran vía s/n, km 2.7 08907 L'Hospitalet, Barcelona, Spain; Fax: + 34932607797; E-mail: 27532dgg@comb.es

On behalf of the Spanish Group of Hemopoietic Transplant (GETH). Received 3 December 2003; accepted 16 January 2004; Published online 18 March 2004 for living patients was 2.8 years (range: 3 months-13 years). All the patients were older than 15 years and conditioned with myeloablative regimens.

Patients with AML beyond first complete remission and $\mathrm{CML}$ beyond first chronic phase were considered as with an advanced phase of disease. We considered sex mismatch as a female donor and a male recipient. GvHD was diagnosed and classified according to the Seattle's criteria. Transplant-related mortality (TRM) was defined as a death without disease relapse.

Univariate analysis for overall survival (OS) and disease-free survival (DFS), as well as the actuarial probabilities for grades IIIIV acute GVHD, TRM and relapse were calculated by the Kaplan and Meier method. Comparison of curves was performed using the log-rank test. Multivariate analysis for OS was performed using the stepwise proportional hazard regression model.

The actuarial OS at 8 years was $59.2 \%$ for the whole cohort of patients. The multivariate analysis detected only three independent risk factors for poor survival: Age beyond 30 years $(P=0.003$, OR: $2.40 ; 95 \% \mathrm{Cl}: 1.34-4.30)$, advanced phase of disease $(P<0.001$, OR: 3.03; 95\% Cl: 1.92-4.78) and female donor for a male recipient $(P=0.023$, OR: $1.71 ; 95 \% \mathrm{Cl}: 1.07-$ 2.74). Based on the results obtained in the multivariate analysis, patients were divided in four groups (or scores) depending on the following criteria: score 1: no risk factors (age $<30$ years, early phase of disease and absence of sex mismatch), 58 cases; score 2: early phase of disease with 1 or 2 risk factors (age $>30$ years \pm sex mismatch), 182 cases; score 3: advanced disease \pm age beyond 30 years or sex mismatch, 54 cases; and score 4: all three risk factors, 22 cases.

Figure 1 shows the actuarial OS and DFS for each score. The actuarial OS was 94.2, 59.1, 45.7 and $8.3 \%$ for patients with scores $1-4$, respectively. These differences were statistically significant (score 1 vs score 2: $P<0.001$; score 2 vs score 3: $P=0.001$; score 3 vs score $4: P=0.010$ ). Actuarial DFS was $72.9,51.6,29.9$ and $0 \%$ for each group. The comparison between each score was also statistically significant (score 1 vs 


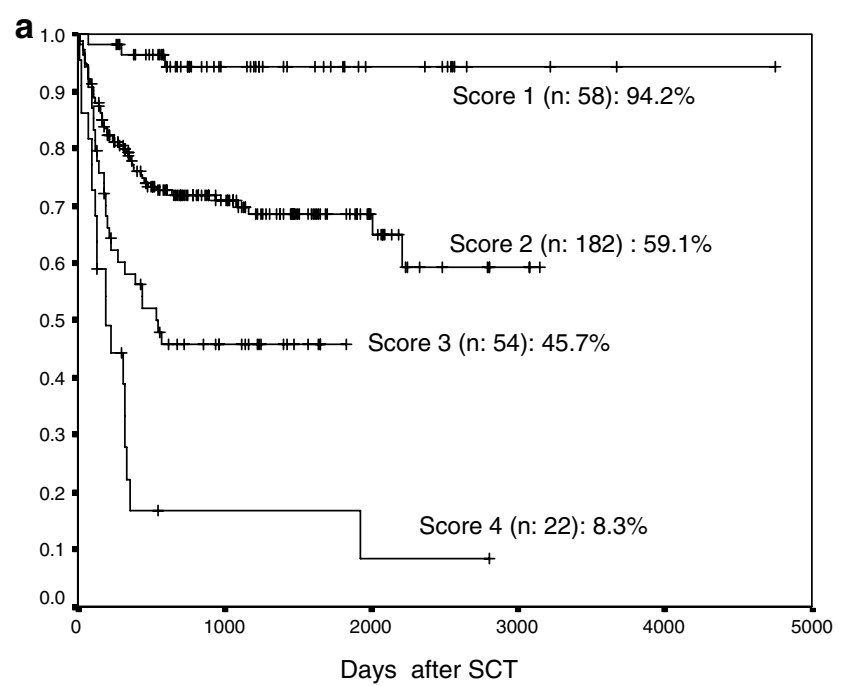

b

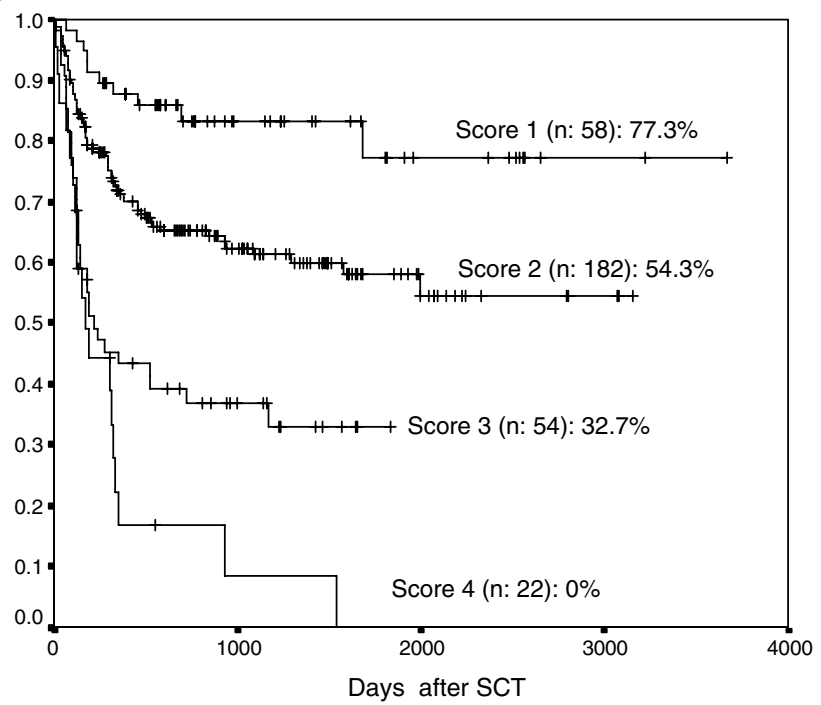

Figure 1 (a) OS actuarial curves depending on the score. (b) DFS on the basis of score.

score 2: $P=0.004$; score 2 vs score 3: $P<0.001$; score 3 vs score 4: $P=0.063)$. When considering the TRM probability, there was a significant increase when comparing patients with score 1 $(1.8 \%)$ and score $2(19.5 \%)(P=0.002)$ and patients with score 3 $(14 \%)$ and score $4(75.7 \%)(P<0.001)$. Actuarial probability of developing grades III-IV acute GvHD was 10.6, 17.4, 22.8 and $33.7 \%$ for scores $1-4$, respectively. The relapse rate for each score was 21.3, 34.9, 61.1 and $100 \%$. Figure 2 shows the actuarial curves for OS and DFS separately for patients with $\mathrm{AML}$ or CML. The differences in OS between scores 1-4 becomes more evident in AML patients: 91.7, 61, 39.3 and 8.3\% for scores $1-4$ (score 1 vs score 2: $P=0.003$; score 2 vs score 3: $P=0.016$; score 3 vs score $4: P=0.003)$. DFS was also clearly influenced by the score in AML patients: 80.7, 56.3, 27.3 and $7.1 \%$ for scores $1-4$ respectively.

We have identified two previously described risk factors for acute GvHD (sex mismatch and patient older than 30 years) ${ }^{1,2}$ as independent risk factors for OS and DFS, increasing the risk of transplant-related death. Older age of the patient is also associated to an increased number of toxic deaths due to the conditioning regimen. The advanced phase of the disease is the third identified

risk factor associated with a lower survival. Obviously, this is due to the higher incidence of relapse in these patients. We therefore developed a scoring system to predict the outcome of the allogeneic transplantation for a given patient, which may be useful to offer to the patient a realistic expected survival after SCT. This approach has been used before to assess the risk of hematologic malignancies, such as non-Hodgkin's lymphoma. ${ }^{3}$

The Chronic Leukemia Working Party of the EBMT published a similar scoring approach for patients with CML receiving a stem cell donor. ${ }^{4}$ They identified the same three pretransplant risk factors (disease stage, age of the patient and sex combination), but they added other two variables: donor type (related or unrelated donor transplant) and time from diagnosis to transplantation (before or after 12 months), identifying seven groups, with an OS of $72,70,62,48,40,18$ and $22 \%$ for patients with score 0-6, respectively. More recently, the Acute Leukemia Working Party of the EBMT published a similar experience when considering patients with acute lymphoblastic leukemia transplanted from an HLA-identical sibling donor. ${ }^{5}$ For these patients, three risk factors were considered: patient's age $>35$ years old, first remission achievement with one or more induction courses, and the male recipient/female donor sex combination.

Our work is the first clinical observation applying a prognostic score to identify patients with AML who benefit most from allogeneic hematopoietic stem cell transplantation.

Obviously, patients in advanced phase of the disease have a higher risk of death. However, OS may improve if we select an HLA-identical sibling donor without sex mismatch, because patients with score 3 have a chance of about $45 \%$ to be cured after allogeneic SCT, whereas survival for those with score 4 is under $10 \%$. It is worth mentioning the absence of statistically significant differences between score 2 and 3 on OS for patients with $\mathrm{CML}$. This effect may be due to the better control of $\mathrm{CML}$ relapses after allogeneic SCT. ${ }^{6}$

This grading score system allows the identification of four risk groups for patients with AML receiving an allogeneic SCT from an HLA-identical sibling donor based on pre-SCT variables easily available. This score could be used to improve the results of the poor-prognosis scores with innovative clinical trials. However, the main benefit of this classification is the possibility to offer a realistic percentage of success to the patient before the starting of the procedure, an information that is essential when discussing the transplant risk for the patient's informed consent.

\section{Acknowledgements}

This study has been financed in part by Grant FIS PI020148 from the Fondo de Investigaciones Sanitarias, Instituto de Salud Carlos III. Ministerio de Sanidad y Consumo.

D Gallardo ${ }^{1}$

R De la Cámara ${ }^{1}$

A Torres ${ }^{1}$

S Brunet ${ }^{1}$

A Jiménez ${ }^{1}$

JC Vallejo ${ }^{1}$

G Sanz ${ }^{1}$

D Serrano ${ }^{1}$

E Carreras $^{1}$

C Martín ${ }^{1}$

C Sanz-Rodríguez ${ }^{1}$

J Sierra ${ }^{1}$

I Espigado ${ }^{1}$

D Caballero ${ }^{1}$

Jj Berlanga ${ }^{1}$
${ }^{1}$ Spanish Group of Hemopoietic Transplant (GETH) 
a

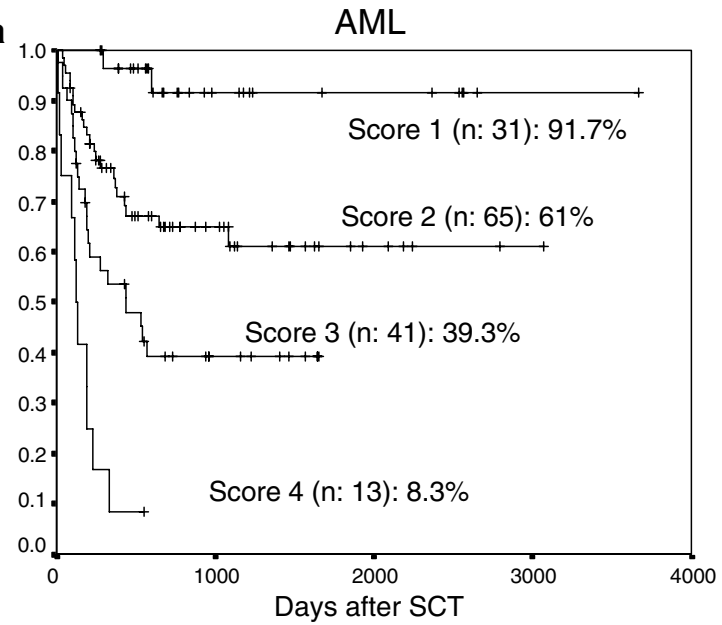

b

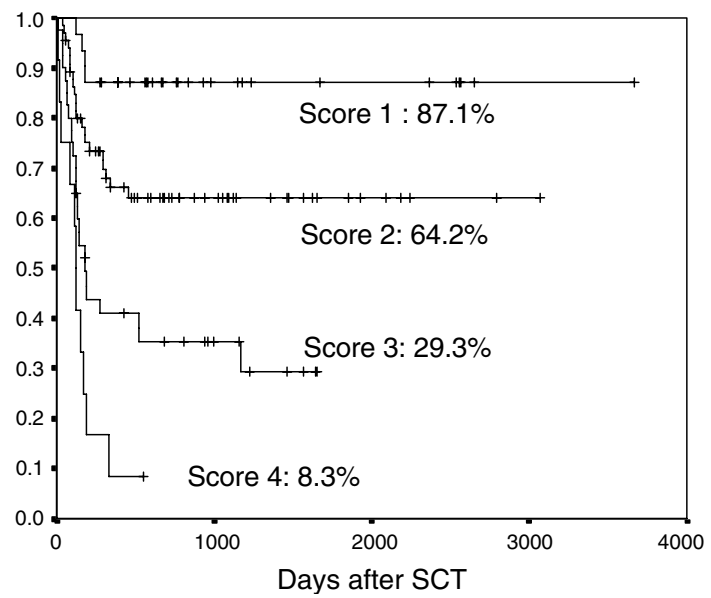

CML
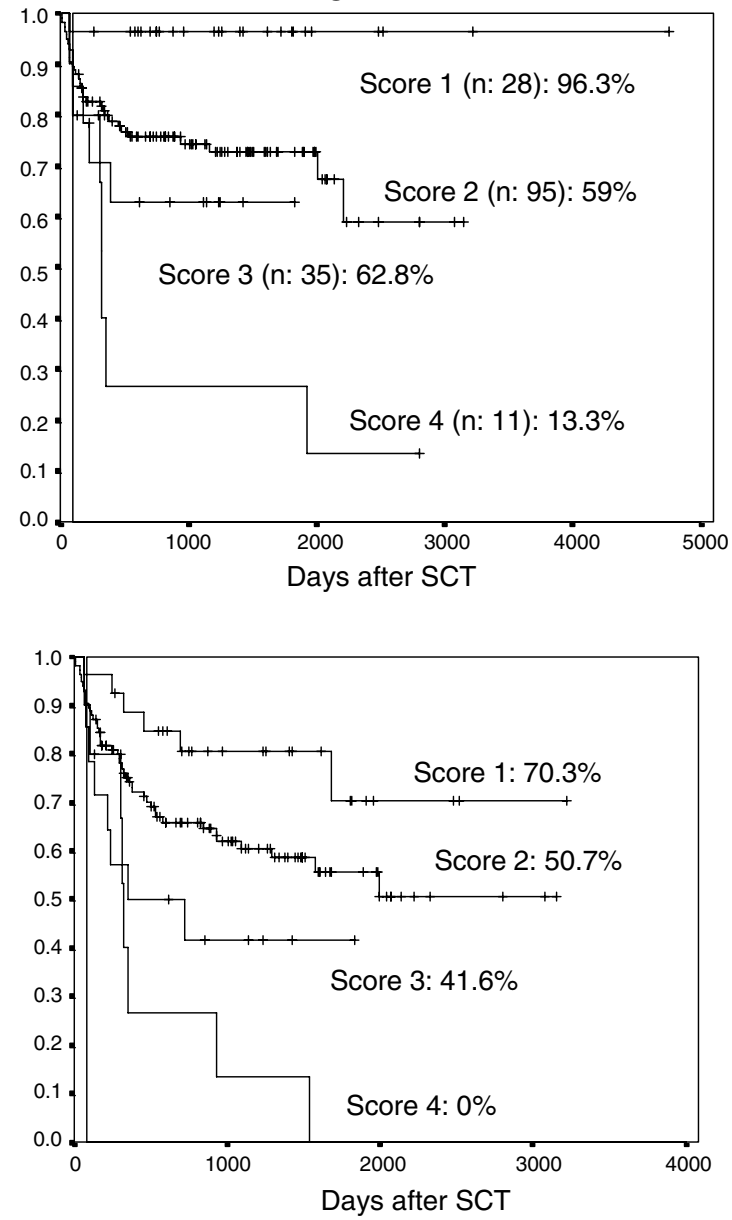

Figure 2 (a) OS for AML patients and for CML patients depending on the score. (b) DFS for AML (left) and CML (right) patients.

\section{References}

1 Gale RP, Bortin MM, van Bekkum DW, Biggs JC, Dicke KA, Gluckman $\mathrm{E}$ et al. Risk factors for acute graft-versus-host disease. Br J Haematol 1987; 67: 397-406.

2 Nash RA, Pepe MS, Storb R, Longton G, Pettinger M, Anasetti C et al. Acute graft-versus-host disease: analysis of risk factors after allogeneic marrow transplantation and prophylaxis with cyclosporine and methotrexate. Blood 1992; 80: 1838-1845.

3 The international non-Hodgkin's lymphoma prognostic factors project. A predictive model for the aggressive non-Hodgkin's lymphoma. N Eng J Med 1993; 329: 987-994.

4 Gratwohl A, Hermans J, Goldman JM, Arcese W, Carreras E, Devergie A et al. Risk assessment for patients with chronic myeloid leukaemia before allogeneic blood or marrow transplantation. Lancet 1998; 52: 1087-1092.

5 Gorin NC, Labopin M, Polge E, Cordonnier C, Jouet JP, Michallet $M$ et al. Risk assessment in adult acute lymphoblastic leukaemia before early haemopoietic stem cell transplantation with a geno-identical donor: an easy clinical prognostic score to identify patients who benefit most from allogeneic haemopoietic stem cell transplantation. Leukemia 2003; 17: 1596-1599.

6 Kolb HJ, Schattenberg A, Goldman J, Hertenstein B, Jacobsen N, Arcese $\mathrm{W}$ et al. Graft-versus-leukemia effect of donor lymphocyte transfusions in marrow grafted patients. European Group for Blood and Marrow Transplantation Working Party Chronic Leukemia. Blood 1995; 86: 2041-2050. 